\title{
Frameless Robot-Assisted Stereotactic Biopsies for Lesions of the Brainstem - A Series of 103 Consecutive Biopsies
}

Iulia Peciu-Florianu ( $\nabla$ iuliapeciu_florianu@yahoo.com )

CHRU de Lille: Centre Hospitalier Universitaire de Lille https://orcid.org/0000-0002-6734-5105

\section{Victor Legrand}

CHRU de Lille: Centre Hospitalier Universitaire de Lille

Apolline Monfilliette-Djelad

CHRU de Lille: Centre Hospitalier Universitaire de Lille

Claude-Alain Maurage

CHRU de Lille: Centre Hospitalier Universitaire de Lille

\section{Quentin Vannod-Michel}

CHRU de Lille: Centre Hospitalier Universitaire de Lille

\section{Serge Blond}

CHRU de Lille: Centre Hospitalier Universitaire de Lille

\section{Gustavo Touzet}

CHRU de Lille: Centre Hospitalier Universitaire de Lille

Nicolas Reyns

CHRU de Lille: Centre Hospitalier Universitaire de Lille

\section{Research Article}

Keywords: brainstem, frameless, robot, stereotactic, biopsy, case series

Posted Date: December 3rd, 2021

DOI: https://doi.org/10.21203/rs.3.rs-1127767/v1

License: (c) (1) This work is licensed under a Creative Commons Attribution 4.0 International License. Read Full License

Version of Record: A version of this preprint was published at Journal of Neuro-Oncology on January 27th, 2022. See the published version at https://doi.org/10.1007/s11060-022-03952-6. 


\section{Abstract}

\section{Purpose}

Targeted treatment for brainstem lesions requires above all a precise histopathological and molecular diagnosis. In the current technological era, robot-assisted stereotactic biopsies represent an accurate and safe procedure for tissue diagnosis.

We present our center's experience in frameless robot-assisted biopsies for brainstem lesions.

\section{Methods}

We performed a retrospective analysis of all patients benefitting from a frameless robot-guided stereotactic biopsy at our University Hospital, from 2001 to 2017. Patients consented to the use of data and/or images. The NeuroMate ${ }^{\circledR}$ robot (Renishaw ${ }^{\mathrm{TM}}$, UK) was used. We report on lesion location, trajectory strategy, histopathological diagnosis and procedure safety.

\section{Results}

Our series encompasses 96 patients (103 biopsies) treated during a 17 years period. Mean age at biopsy: 34.0 years (range 1-78). Most common location: pons (62.1\%). Transcerebellar approach: 61 procedures (59.2\%). Most common diagnoses: diffuse glioma (67.0\%), metastases (7.8\%) and lymphoma (6.8\%). Non conclusive diagnosis: 10 cases (9.7\%). After second biopsy this decreased to 4 cases $(4.1 \%)$. Overall biopsy diagnostic yield: $95.8 \%$. Permanent disability was recorded in 3 patients (2.9\%, all adults), while transient complications in 17 patients (17.7\%). Four cases of intra-tumoral hematoma were recorded (one case with rapid decline and fatal issue). Adjuvant targeted treatment was performed in $72.9 \%$ of patients. Mean follow-up (in the Neurosurgery Department): 2.2 years.

\section{Conclusion}

Frameless robot-assisted stereotactic biopsies can provide the initial platform towards a safe and accurate management for brainstem lesions, offering a high diagnostic yield with low permanent morbidity.

\section{Introduction}

Brainstem lesions are a rare and very heterogeneous group of tumors, comprising $10 \%$ of brain tumors in the pediatric population[1] and $1-2 \%$ in adults[2]. Specific multimodal treatment for these lesions remains challenging, even in the current era of consistent progress in microsurgery and therapeutic advances. A precise histopathological and molecular diagnosis is thus the first step towards a targeted therapy.

Surgical robotics represent an important technological innovation[3], increasing the possibility of accurate and safe procedures. Used in a variety of surgical specialties robotics have integrated the 
surgeon's armamentarium, opening new avenues for diagnostics and treatment. In neurosurgery robotics have been implemented to improve a range of stereotactic procedures, such as deep brain stimulation[4], stereo-electroencephalography (SEEG)[5], intracranial catheter placement[6] or high accuracy biopsies[7].

Stereotactic brainstem biopsies have been described as an alternative to open biopsies for these deeplocated lesions, either using frame-based[8-10] of frameless systems[11, 12]. Robot-assisted stereotactic biopsies allow a supplementary increase in accurate sampling and increase in technical ease and safety[7, 13-16].

We present our center's experience in performing frameless robot-assisted biopsies for lesions of the brainstem.

\section{Methods}

\section{Study design and patient population}

We performed a retrospective analysis of all consecutive patients benefitting from a frameless robotguided stereotactic biopsy at our University Hospital, from January 2001 to December 2017. This allowed the recovery of records for an extended follow-up period.

Included cases presented with a brainstem lesion of unknown entity, requiring a biopsy according to a multidisciplinary neuro-oncology board discussion.

Brainstem lesions were defined on prior diagnostic cerebral MRI as involving the mesencephalon (tectum or tegmentum), crus cerebri, pons or medulla oblongata. Lesions occurring in the middle cerebellar peduncle, extending into the cerebellum or in the diencephalon were excluded. There were no exclusion criteria based on age.

All patients have given written informed consent for the procedure and use of anonymized data and/or images. This research study was conducted retrospectively from data obtained for clinical purposes. We consulted extensively with the IRB of College de Neurochirurgie who determined that our study meet the criteria for ethical approval: $n^{\circ}$ IRB00011687. This case series has been reported in line with the PROCESS Guideline[17].

\section{Frameless robot-assisted biopsy technique}

The Neuromate ${ }^{\circledR}$ robot (Renishaw, Gloucestershire, UK) was used in all cases. Complete procedure consisted of four stages: fiducial marker placement, cerebral MRI, trajectory planning and frameless robot-assisted stereotactic biopsy.

Fiducial marker placement was performed under local anesthesia in all adult patients. General anesthesia was used for the entire procedure in pediatric patients. Optimal fiducial marker placement requiring a minimal bone fixation was decided according to lesion location and laterality. 
A T1-weighted 3D Gadolinium-enhanced thin-sliced $(\leq 1 \mathrm{~mm})$ cerebral MRI was acquired, using a mounted microphone satellite on the fiducial marker, associated, if necessary, with other MRI sequences.

Trajectory planning was performed after image transfer towards the Neuromate ${ }^{\circledR}$ robot's computer, using the VoXim ${ }^{\circledR}$ program. Optimal target depended on tumor borders, neighboring anatomic structures (vascular or functional brainstem areas) and lesion enhancement. Final trajectory was planned in double obliquity (trajectory axis from anterior to posterior and from lateral to medial), either transcerebellar (Figure 1) in lesions extending from the medulla and in the majority of pontine lesions, while the transfrontal (Figure 2) intraparenchymal or transventricular was chosen in mesencephalic or pontine lesions extending in the mesencephalon (bi-compartmental lesions).

Biopsy procedure was performed under general anesthesia in all patients, using the Neuromate ${ }^{\circledR}$ headpin fixation system (Figure 3). Correlation between patient position and the robotic arm was achieved using the ultrasound emitter tool specific to this device and the fiducial cranial marker previously placed. A laser verification trajectory was performed to verify accuracy of coregistration. Biopsy was performed in a step-wise fashion, with continuous computer input of the Sedan-Valliccioni side-cut needle advancement to the planned target. Specimens were acquired in a circular (rosette) pattern, at different depth levels to acquire pathologic samples for analysis (fresh and in formalin fixated containers).

A biopsy was considered positive if acquired specimens resulted in a diagnosis based on histology analysis and/or molecular markers.

\section{Data acquisition}

A patient database was retrospectively created. Patient files were recovered from the Neurosurgical, Pathology and Neuro-oncology Departments, for complete records concerning demographics, lesion location, trajectory choice, histopathological diagnosis, treatment and follow-up.

All patients biopsied between January 2001 and December 2017 were included in final data analysis. There were no exclusion criteria based on age at the time of biopsy.

\section{Statistical analysis}

Statistical analysis was performed in the Biostatistics Department of our University Hospital, using the Statistical Analysis Software ${ }^{\circledR}$ (SAS Institute Inc., version 9.4). Quantitative variables were described using mean \pm SD (standard deviation) or the median if non-Gaussian distribution was observed. Distribution normalization was verified with the Shapiro-Wilk test. Qualitative variables were described using frequencies and percentages.

Localization, trajectory, complications, diagnosis and biopsy accuracy were compared between the adult and pediatric population using the Chi-square distribution or the Fisher test $(p \leq 0.5)$.

\section{Results}


Our series encompasses 96 patients treated in a 17 years period (January 2001 - December 2017), for a total of 103 biopsies.

\section{Demographics}

Table 1 describes main demographic data. Mean age at biopsy was 34.0 years (range 1-78). Biopsies were performed in 31 pediatric patients ( $<16$ years old, $32.3 \%$ ) and in 65 adults $(67.7 \%)$.

Table 1

Main demographic and clinical presentation data

\begin{tabular}{|c|c|c|c|c|}
\hline Age & $0-16$ years & $\begin{array}{l}17-39 \\
\text { years }\end{array}$ & $40-69$ years & $\geq 70$ years \\
\hline & 31 (32.3\%) & $21(21.8 \%)$ & $40(41.6 \%)$ & $4(4.2 \%)$ \\
\hline Sex & $\mathrm{F} / \mathrm{M}$ & $45 / 49$ & & \\
\hline \multirow[t]{2}{*}{ Location } & Mesencephalon & Pons & $\begin{array}{l}\text { Medulla } \\
\text { oblongata }\end{array}$ & $\begin{array}{l}\text { Brainstem (global } \\
\text { infiltration) }\end{array}$ \\
\hline & 24 & 64 & 1 & 7 \\
\hline \multirow{8}{*}{$\begin{array}{l}\text { Main symptoms } \\
\text { (number, \%) }\end{array}$} & Ataxia & 45 (46.8\%) & & \\
\hline & Motor deficit & $12(12.5 \%)$ & & \\
\hline & $\begin{array}{l}\text { Oculomotor } \\
\text { disturbance }\end{array}$ & $13(13.5 \%)$ & & \\
\hline & Sensory deficit & $6(6.2 \%)$ & & \\
\hline & Dysphagia & $5(5.2 \%)$ & & \\
\hline & $\begin{array}{l}\text { Intracranial } \\
\text { hypertension }\end{array}$ & $9(9.4 \%)$ & & \\
\hline & Asymptomatic & $1(1 \%)$ & & \\
\hline & Other & $5(5.2 \%)$ & & \\
\hline Hydrocephalus & $\mathrm{Y} / \mathrm{N}$ & $\begin{array}{l}19 \\
(19.8 \%) / 77\end{array}$ & & \\
\hline
\end{tabular}

All brainstem locations were included. Main location was determined by the lesion's volume situated in a certain anatomical structure on the preoperative MRI: mesencephalon (tectum or tegmentum), crus cerebri, pons or medulla oblongata. Most common location was the pontine region, in 64 patients (62.1\%), more frequently seen in the pediatric group (23 patients, $74.2 \%$ ) than in the adult group (41 patients, $56.9 \%), p=0.021$. The mesencephalon or/and crus cerebri were involved in 24 patients $(23.3 \%)$. The medulla oblongata was biopsied in only 1 patient (1\%). 
Main symptoms and clinical signs at diagnosis (Table 1) were balance impairment, diplopia, motor deficit and facial paresis. Less frequently dysphagia, dysarthria, sensory disturbance or signs of intracranial hypertension were described. $68 \%$ of patients presented with a combination of three or more symptoms and clinical signs.

\section{Trajectory}

A transcerebellar - trans middle cerebellar peduncle approach was used in 61 patients (59.2\%), with 54 of those patients presenting with a main pontine lesion location. No significant difference was observed between the optimal chosen trajectory in the pediatric versus adult group $(p=0.78)$.

\section{Histopathology and diagnostic precision}

Histopathologic diagnosis was achieved in $95.8 \%$. Second biopsy was needed in 6 patients (6.2\%), with a cerebral MRI showing the initial biopsy target outside the lesion in only two patients.

Various diagnoses are described (Table 2), most commonly being diffuse glioma (68 patients, $70.8 \%$ ), metastases (8 patients, 8.3\%) and lymphoma (7 patients, 7.3\%). Gliomas grade III and IV were the most frequent in their group, with 22 and 26 cases, respectively ( $23 \%$ and $27 \%$, respectively). Overall, gliomas were statistically more frequently diagnosed in the pediatric group ( 26 patients, $83.9 \%, p=0.017$ ). Nine patients $(9.4 \%)$ presented with a rare histopathologic diagnosis for lesions situated in the brainstem: abscess (3 patients, 3.1\%), primitive neuroectodermal tumor (PNET, 2 pediatric patients, $2 \%$ ), cavernoma (1 patient, 1\%), hemorrhagic cyst (1 patient, 1\%), histiocytosis (1 patient, 1\%) and inflammatory lesion (progressive multifocal leukoencephalopathy, 1 patient, $1 \%$ ). 
Table 2

Biopsy related details and histopathology

\begin{tabular}{|c|c|c|}
\hline \multirow[t]{2}{*}{ Trajectory } & Transcerebellar & Transfrontal \\
\hline & 61patients & 42 patients \\
\hline \multicolumn{2}{|c|}{ Postoperative clinical worsening (Yes/No patients, \%) } & $20(19.4 \%) / 83$ \\
\hline \multicolumn{2}{|c|}{ Second biopsy (Yes/No patients, \%) } & $6(6.2 \%) / 90$ \\
\hline \multirow{14}{*}{$\begin{array}{l}\text { Anatomopathology (number of } \\
\text { patients) }\end{array}$} & Glioma grade I & 3 \\
\hline & Glioma grade II & 17 \\
\hline & Glioma grade III & 22 \\
\hline & Glioma grade IV & 26 \\
\hline & \multicolumn{2}{|c|}{$\begin{array}{l}\text { Midline diffuse glioma }(\mathrm{H} 3 \mathrm{~K} 27 \mathrm{M})-7 \text { patients of total glioma } \\
\text { diagnosis }\end{array}$} \\
\hline & Metastases & 8 \\
\hline & Lymphoma & 7 \\
\hline & Abscess & 3 \\
\hline & PNET & 2 \\
\hline & Cavernoma & 1 \\
\hline & Hemorrhagic cyst & 1 \\
\hline & Histiocytosis & 1 \\
\hline & Inflammatory (LEMP) & 1 \\
\hline & Inconclusive result aft & 4 \\
\hline
\end{tabular}

Since the publication of the 2016 WHO Classification of Tumors of the Central Nervous System[18], histopathology was extended to include systematic genetic analysis, especially for histone mutations. This analysis could be performed in 10 cases, revealing 7 cases of diffuse midline glioma, presenting with a H3.3 (K27M or K28M) mutation, all in the pediatric group.

Non conclusive diagnosis was found in 10 cases (9.7\%). After second biopsy this decreased to 4 cases (4.1\%). No statistical difference was found related to lesion localization $(p=0.7)$ or the chosen trajectory (transcerebellar versus transfrontal, $p=0.73$ ).

\section{Biopsies-related morbidity}

Permanent morbidity rate was $2.9 \%$ of all performed biopsies (3 patients). Overall morbidity was recorded in $19.4 \%$ of cases (Table 3 ), concerning worsening of existing symptoms (9 patients, $8.7 \%$ ) or new onset 
symptoms (11 patients, 10.7\%). The most common was oculomotor transitory disorder (10 patients).

Table 3

Biopsy related morbidity

\begin{tabular}{|lll|}
\hline Worsening of exisiting symptoms $(\mathbf{n}=\mathbf{9})$ & Transient & Definitive \\
\hline Oculomotor palsy & 2 & 0 \\
\hline Worsening of motor status (hemiparesis, hemiplegia or facial paresis) & 3 & 1 \\
\hline Ataxia & 2 & 0 \\
\hline Dysphagia & 0 & 1 \\
\hline New onset symptoms $(\mathrm{n}=11)$ & & \\
\hline Oculomotor palsy & 8 & 0 \\
\hline Segmental sensory disturbance & 1 & 0 \\
\hline Hemiplegia & 0 & 1 \\
\hline Anarthria & 1 & 0 \\
\hline
\end{tabular}

All patients presenting with a postoperative complication benefitted from a cerebral scan or MRI, revealing 4 cases of intra-tumoral hematoma (transient symptom worsening in 3 patients).

Of note, one death was recorded at day 10 postoperative in a 47 years-old male, due to an intra-tumoral pontine hematoma with important mass effect (transcerebellar approach, ipsilateral to the bleeding, trajectory in the periphery of the hematoma; histopathology had revealed a glioblastoma).

Statistical analysis revealed no difference between complications arising after a transcerebellar or a transfrontal trajectory $(\mathrm{p}=0.45)$.

\section{Treatment}

Postoperative treatment is detailed in Table 4. 
Table 4

Treatment strategies

\section{Total (96 patients, \%) Adult / Pediatric}

\begin{tabular}{lll} 
Surveillance & $4(4.2 \%)$ & $3 / 1$ \\
Surgery & & \\
\hline Surgery alone & $1(1,0 \%)$ & $1 / 0$ \\
\hline Surgery followed by chemotherapy and/or radiotherapy & $5(5,2 \%)$ & $0 / 5$ \\
\hline Chemotherapy only & $10(10,4 \%)$ & $9 / 1$ \\
\hline Radiotherapy only & $30(31,2 \%)$ & $23 / 7$ \\
\hline Combined radio-chemotherapy & $30(31,2 \%)$ & $19 / 11$ \\
\hline Palliative care & $7(7,3 \%)$ & $5 / 2$ \\
\hline Targeted antibiotics & $2(2,1 \%)$ & $2 / 0$ \\
\hline Corticotherapy only & $4(4,2 \%)$ & $3 / 1$ \\
\hline NA & $3(3,1 \%)$ & $0 / 3$ \\
\hline
\end{tabular}

Adjuvant targeted treatment was performed in 75 patients ( $78.1 \%$ of patients). Surgery for partial or subtotal resection was possible in 6 patients (one adult, five pediatric patients, after adjuvant therapy in five cases), all low-grade gliomas (grade I or II WHO), deemed operable due to the presence of an exophytic component. All surgical approaches were performed in our Neurosurgical Department using either a subtemporal transtentorial approach for mesencephalic lesions (4 patients) or a suboccipital telovelar approach for pontine lesions (2 patients).

\section{Follow-up}

Mean follow-up in the Neurosurgery Department was 2.2 years.

The main endpoint of this study is centered on the impact of frameless robot-assisted biopsies in precision diagnosis and global patient management. Overall survival did not thus represent a secondary endpoint in this study.

\section{Discussion}

Robot-assisted surgery allows accurate and safe interventions, often reducing postoperative risks and shortening burdensome recovery periods. In neurosurgery, robotics expanded the depth of stereotactic and functional neurosurgery, integrating the team as a tool of utmost importance.

Biopsy procedures have been a constant part of the neurosurgical armamentarium, constantly evolving in the last decades. Even more so in the subtle field of brainstem lesions that historically were considered 
mostly inoperable and related to significant morbidity[19]. In the era of microsurgical techniques, these lesions remain challenging, requiring a precise approach through safe entry zones, in meticulously selected cases [20].

Robot-assisted biopsies can increase the potential of targeting eloquent deep-seated locations with lowered risk of complications and high diagnostic yield. Optimal trajectory planning takes into consideration lesion location, traversing grey and white matter, vessels but also vascular structures (pia layer, ependymal cells, choroid plexus) and ventricle avoidance[21-23].

\section{Routes to the brainstem}

Brainstem lesions have always generated debates from operability to adequate management. In a quest to minimize morbidity safe passageways have been methodically described from the microsurgical point of view, encompassing multiple supra and infratentorial approaches [20, 24-26]. Two stereotactic safe routes to the brainstem have proven their versatility to reach intrinsic brainstem lesions and deserve special consideration: the transcerebellar and the transfrontal routes.

The most frequently used is the transcerebellar approach, automatically linked in our opinion to the more frequent localization of lesions in or extending towards the pontine region. This approach implies an entry point on the surface of the cerebellar hemispheres, navigating the height and width of the middle cerebellar peduncle to reach mainly pontine lesions but also low-hanging mesencephalic or higher bulbar lesions. In our study, this approach was used in $59.2 \%$ of cases.

The transcerebellar approach has been described using a stereotactic frame $[8,10,27,28]$ or frameless $[13,15]$, with favorable results concerning complications, varying from $0 \%$ (in small cohorts) to $19.2 \%$ for transitory morbidity. Main advantages are related to shorter trajectories, passing through the middle cerebellar peduncle, thus lowering the theoretical risk of hemorrhage. In our study $16.4 \%$ of patients (10/66 cases) benefitting from a transcerebellar approach presented a biopsy-related complication, most common transitory balance impairment or motor status worsening. One noteworthy technical advantage of using a stereotactic robot regards the easiness of patient positioning, independent to frame adaptations described for brainstem lesions[28].

This versatile approach is complemented by the transfrontal approach, especially for mesencephalic or rostral pontine lesions. The transfrontal route is planned frequently from a precoronal entry point, navigating between the lateral ventricle, head of the caudate, basal ganglia, and internal capsule (Figure $2 \mathrm{~B}$ and $2 \mathrm{C}$ ). Despite longer trajectories, the transfrontal approach remains a viable option, with a low rate of complications (in our study, most often diplopia or motor status worsening)[21, 29].

Our study shows no statistical difference in biopsy-related morbidity between the transfrontal and transcerebellar trajectories $(p=0.45)$. In their meta-analysis, Kickingereder et al.[30] report that the weighted average proportion calculated by random-effects was 7.8\% (95\% Cl: $5.6 \%-10.2 \%)$ for overall morbidity, $1.7 \%$ (95\% Cl: 0.9\%-2.7\%) for permanent morbidity, and $0.9 \%$ (95\% Cl: 0.5\%-1.4\%) for mortality. 
In an updated meta-analysis for pediatric brainstem tumors, Hamisch et al.[31] report similar results: $6.7 \%$ (95\% Cl 4.2-9.6\%) for overall morbidity, 0.6\% (95\% Cl 0.2\%- 1.4\%) for permanent morbidity, and $0.6 \%$ $(95 \% \mathrm{Cl} 0.2 \%-1.3 \%)$ for mortality. Both studies showed no significant differences between the transcerebellar and transfrontal routes to the brainstem.

\section{Impact in molecular diagnosis evolution}

In our retrospective study, histopathological diagnosis was achieved in $95.8 \%$ of cases, similar to available data. A second biopsy was necessary in 6 patients. Kickingereder et al.[30] reported on 1480 cases of frame-based or frameless stereotactic brainstem biopsies, comprising 38 studies, with a final diagnosis achieved in $96.2 \%$. Interestingly, the authors underlined the need for highly specialized centers to increase diagnostic success, with a significant correlation between diagnostic rates and the number of biopsies performed annually in each center $(p=0.011)$. Additionally, Hamisch et al.[31] reported in their meta-analysis on 18 studies a diagnostic rate of $96.1 \%$ in a total of 735 pediatric brainstem biopsies.

Our center's philosophy is to biopsy all brainstem tumors before discussing appropriate adjuvant treatment. In the era of integrated molecular analysis stereotactic brainstem biopsies could assist in early initiation of targeted therapies, especially in the diffuse intrinsic pontine glioma (DIPG) subgroup with the analysis of histone mutations (e.g., H3.3 K27M)[32]. This has marked an important change in the treatment paradigm of brainstem lesions, from the initial concept of MRI exclusive diagnosis[33] towards precise molecular diagnosis[34-36] and current targeted treatment[37, 38].

Noteworthy, our study did not allow statistical analysis based on molecular diagnosis, covering a long period prior to the integration of molecular biology data.

\section{Differential diagnosis for brainstem tumors}

In our study we report 68 cases of diffuse glioma, representing $70.8 \%$ of the total number of successful biopsies. Brainstem metastasis was confirmed in 8 patients (8.3\%) while lymphoma was reported in 7 patients (7.3\%). Noteworthy, 9 patients presented with a rare histopathologic diagnosis (Table 2), illustrating the difficulty of radiologic diagnosis solely on cerebral MRI, thus warranting a stereotactic biopsy even in non-tumoral pathologies.

As reported by several studies, a step-wise MRI-based approach could help differentiate between several etiologies, especially tumor-like mimics: infectious and inflammatory lesions, vascular lesions or even uncommon cysts[39-41]. While morphology details can be quickly helpful in some pathologies, differential diagnosis of immune-mediated and inflammatory lesions of the brainstem can remain as elusive as ever. Law et al. proposed integrating essential clinical, biological and radiological data into the differential diagnosis, reviewing a remarkable large spectrum of these disorders based on cerebral MRI findings[39]. Routine cranio-spinal morphology MRI sequences could be coupled with more advanced modalities: PET-CT, MRI spectroscopy and MRI/CT perfusion scans[39, 40, 42].

\section{Limitations}


We acknowledge several limitations in our study. Firstly, it is a retrospective series with data acquisition limited by the 17 years period encompassed in our analysis. Secondly, while it is a monocentric study, patients were referred from other neurosurgical centers and thus were sometimes lost early at follow-up. This was nonetheless not the main focus in our analysis, permitting the inclusion of all consecutive patients for final analysis. Thirdly, histopathology results were limited by the absence of systematic genetic testing prior to the 2016 WHO Classification, limiting our insight into a developing and promising avenue for clinical research.

\section{Conclusion}

Frameless robot-assisted stereotactic biopsies can provide the initial platform towards a safe and accurate management for brainstem lesions. This single center series focuses on high diagnostic yield for frameless robot-assisted stereotactic biopsies, complemented by low permanent morbidity.

\section{Declarations}

\section{Funding}

The authors declare that no funds, grants, or other support were received during the preparation of this manuscript.

\section{Competing Interests}

The authors have no relevant financial or non-financial interests to disclose.

\section{Author Contributions}

All authors contributed to the study conception and design. Material preparation, data collection and analysis were performed by lulia Peciu-Florianu and Victor Legrand. The first draft of the manuscript was written by lulia Peciu-Florianu and all authors commented on previous versions of the manuscript. All authors read and approved the final manuscript.

\section{Data Availability}

The datasets generated during and/or analysed during the current study are available from the corresponding author on reasonable request.

\section{Ethics Approval}

This research study was conducted retrospectively from data obtained for clinical purposes. We consulted extensively with the IRB of College de Neurochirurgie who determined that our study meet the criteria for ethical approval: n IRB00011687. 
Informed consent was obtained from all individual participants included in the study.

\section{References}

1. Johnson, K.J., et al., Childhood brain tumor epidemiology: a brain tumor epidemiology consortium review. Cancer Epidemiol Biomarkers Prev, 2014. 23(12): p. 2716-36.

2. Ostrom, Q.T., et al., CBTRUS Statistical Report: Primary Brain and Central Nervous System Tumors Diagnosed in the United States in 2008-2012. Neuro Oncol, 2015. 17 Suppl 4: p. iv1-iv62.

3. Hughes-Hallett, A., et al., Quantifying innovation in surgery. Ann Surg, 2014. 260(2): p. 205-11.

4. Ughratdar, I., M. Samuel, and K. Ashkan, Technological Advances in Deep Brain Stimulation. J Parkinsons Dis, 2015. 5(3): p. 483-96.

5. Gonzalez-Martinez, J., et al., Technique, Results, and Complications Related to Robot-Assisted Stereoelectroencephalography. Neurosurgery, 2016. 78(2): p. 169-80.

6. Pillai, A., et al., Expanding the Spectrum of Robotic Assistance in Cranial Neurosurgery. Oper Neurosurg (Hagerstown), 2019. 17(2): p. 164-173.

7. Marcus, H.J., et al., Robot-assisted stereotactic brain biopsy: systematic review and bibliometric analysis. Childs Nerv Syst, 2018. 34(7): p. 1299-1309.

8. Spiegelmann, R. and W.A. Friedman, Stereotactic suboccipital transcerebellar biopsy under local anesthesia using the Cosman-Roberts-Wells frame. Technical note. J Neurosurg, 1991. 75(3): p. 4868.

9. Bahrami, E., et al., An Experience with Frame-Based Stereotactic Biopsy of Posterior Fossa Lesions via Transcerebellar Route. World Neurosurg, 2020.

10. Quick-Weller, J., et al., Stereotactic biopsy of brainstem lesions: 21 years experiences of a single center. J Neurooncol, 2016. 129(2): p. 243-50.

11. Coca, H.A., et al., Diffuse intrinsic pontine gliomas in children: Interest of robotic frameless assisted biopsy. A technical note. Neurochirurgie, 2016. 62(6): p. 327-331.

12. Williams, J.R., et al., Progress in diffuse intrinsic pontine glioma: advocating for stereotactic biopsy in the standard of care. Neurosurg Focus, 2020. 48(1): p. E4.

13. Haegelen, C., et al., Stereotactic robot-guided biopsies of brain stem lesions: Experience with 15 cases. Neurochirurgie, 2010. 56(5): p. 363-7.

14. Carai, A., et al., Robot-Assisted Stereotactic Biopsy of Diffuse Intrinsic Pontine Glioma: A SingleCenter Experience. World Neurosurg, 2017. 101: p. 584-588.

15. Dawes, W., et al., Robot-assisted stereotactic brainstem biopsy in children: prospective cohort study. J Robot Surg, 2019. 13(4): p. 575-579.

16. Gupta, M., et al., Robot-assisted stereotactic biopsy of pediatric brainstem and thalamic lesions. J Neurosurg Pediatr, 2020: p. 1-8. 
17. Agha, R.A., et al., The PROCESS 2020 Guideline: Updating Consensus Preferred Reporting Of CasESeries in Surgery (PROCESS) Guidelines. Int J Surg, 2020. 84: p. 231-235.

18. Louis, D.N., et al., The 2016 World Health Organization Classification of Tumors of the Central Nervous System: a summary. Acta Neuropathol, 2016. 131(6): p. 803-20.

19. Buchanan, D., Intracranial tumors in infancy and childhood. Am J Surg, 1957. 93(6): p. 935-40.

20. Cavalcanti, D.D., et al., Microsurgical anatomy of safe entry zones to the brainstem. J Neurosurg, 2016. 124(5): p. 1359-76.

21. Chen, S.Y., et al., Stereotactic biopsy for brainstem lesion: comparison of approaches and reports of 10 cases. J Chin Med Assoc, 2011. 74(3): p. 110-4.

22. Hood, T.W., et al., Stereotaxic biopsy of intrinsic lesions of the brain stem. J Neurosurg, 1986. 65(2): p. 172-6.

23. Amundson, E.W., M.J. McGirt, and A. Olivi, A contralateral, transfrontal, extraventricular approach to stereotactic brainstem biopsy procedures. Technical note. J Neurosurg, 2005. 102(3): p. 565-70.

24. Cavalcanti, D.D., et al., Supracerebellar Infratentorial Variant Approaches to the Intercollicular Safe Entry Zone. World Neurosurg, 2019. 122: p. e1285-e1290.

25. Recalde, R.J., E.G. Figueiredo, and E. de Oliveira, Microsurgical anatomy of the safe entry zones on the anterolateral brainstem related to surgical approaches to cavernous malformations. Neurosurgery, 2008. 62(3 Suppl 1): p. 9-15; discussion 15-7.

26. Kyoshima, K., et al., A study of safe entry zones via the floor of the fourth ventricle for brain-stem lesions. Report of three cases. J Neurosurg, 1993. 78(6): p. 987-93.

27. Quick-Weller, J., et al., Oblique Positioning of the Stereotactic Frame for Biopsies of Cerebellar and Brainstem Lesions. World Neurosurg, 2016. 86: p. 466-9.

28. Capitanio, J.F., et al., Inverted positioning of Leksell Frame $G$ for very low posterior fossa and brain stem lesions biopsies. J Neurosurg Sci, 2019. 63(2): p. 194-199.

29. Goncalves-Ferreira, A.J., M. Herculano-Carvalho, and J. Pimentel, Stereotactic biopsies of focal brainstem lesions. Surg Neurol, 2003. 60(4): p. 311-20; discussion 320.

30. Kickingereder, P., et al., Diagnostic value and safety of stereotactic biopsy for brainstem tumors: a systematic review and meta-analysis of 1480 cases. Neurosurgery, 2013. 72(6): p. 873-81; discussion 882; quiz 882.

31. Hamisch, C., et al., Update on the diagnostic value and safety of stereotactic biopsy for pediatric brainstem tumors: a systematic review and meta-analysis of 735 cases. J Neurosurg Pediatr, 2017. 20(3): p. 261-268.

32. Gupta, N., et al., Prospective feasibility and safety assessment of surgical biopsy for patients with newly diagnosed diffuse intrinsic pontine glioma. Neuro Oncol, 2018. 20(11): p. 1547-1555.

33. Albright, A.L., et al., Magnetic resonance scans should replace biopsies for the diagnosis of diffuse brain stem gliomas: a report from the Children's Cancer Group. Neurosurgery, 1993. 33(6): p. 1026-9; discussion 1029-30. 
34. Louis, D.N., et al., cIMPACT-NOW (the consortium to inform molecular and practical approaches to CNS tumor taxonomy): a new initiative in advancing nervous system tumor classification. Brain Pathol, 2017. 27(6): p. 851-852.

35. Louis, D.N., et al., cIMPACT-NOW update 2: diagnostic clarifications for diffuse midline glioma, H3 K27M-mutant and diffuse astrocytoma/anaplastic astrocytoma, IDH-mutant. Acta Neuropathol, 2018. 135(4): p. 639-642.

36. Louis, D.N., et al., CIMPACT-NOW update 6: new entity and diagnostic principle recommendations of the cIMPACT-Utrecht meeting on future CNS tumor classification and grading. Brain Pathol, 2020. 30(4): p. 844-856.

37. Aziz-Bose, R. and M. Monje, Diffuse intrinsic pontine glioma: molecular landscape and emerging therapeutic targets. Curr Opin Oncol, 2019. 31(6): p. 522-530.

38. Bush, N.A., S.M. Chang, and M.S. Berger, Current and future strategies for treatment of glioma. Neurosurg Rev, 2017. 40(1): p. 1-14.

39. Law, L.Y., et al., The spectrum of immune-mediated and inflammatory lesions of the brainstem: Clues to diagnosis. Neurology, 2019. 93(9): p. 390-405.

40. Haralur, Y. and L.L. Mechtler, Neuroimaging of Multiple Sclerosis Mimics. Neurol Clin, 2020. 38(1): p. 149-170.

41. Prakkamakul, S., et al., MRI Patterns of Isolated Lesions in the Medulla Oblongata. J Neuroimaging, 2017. 27(1): p. 135-143.

42. Gass, A., M. Filippi, and R.I. Grossman, The contribution of MRI in the differential diagnosis of posterior fossa damage. J Neurol Sci, 2000. 172 Suppl 1: p. S43-9.

\section{Figures}




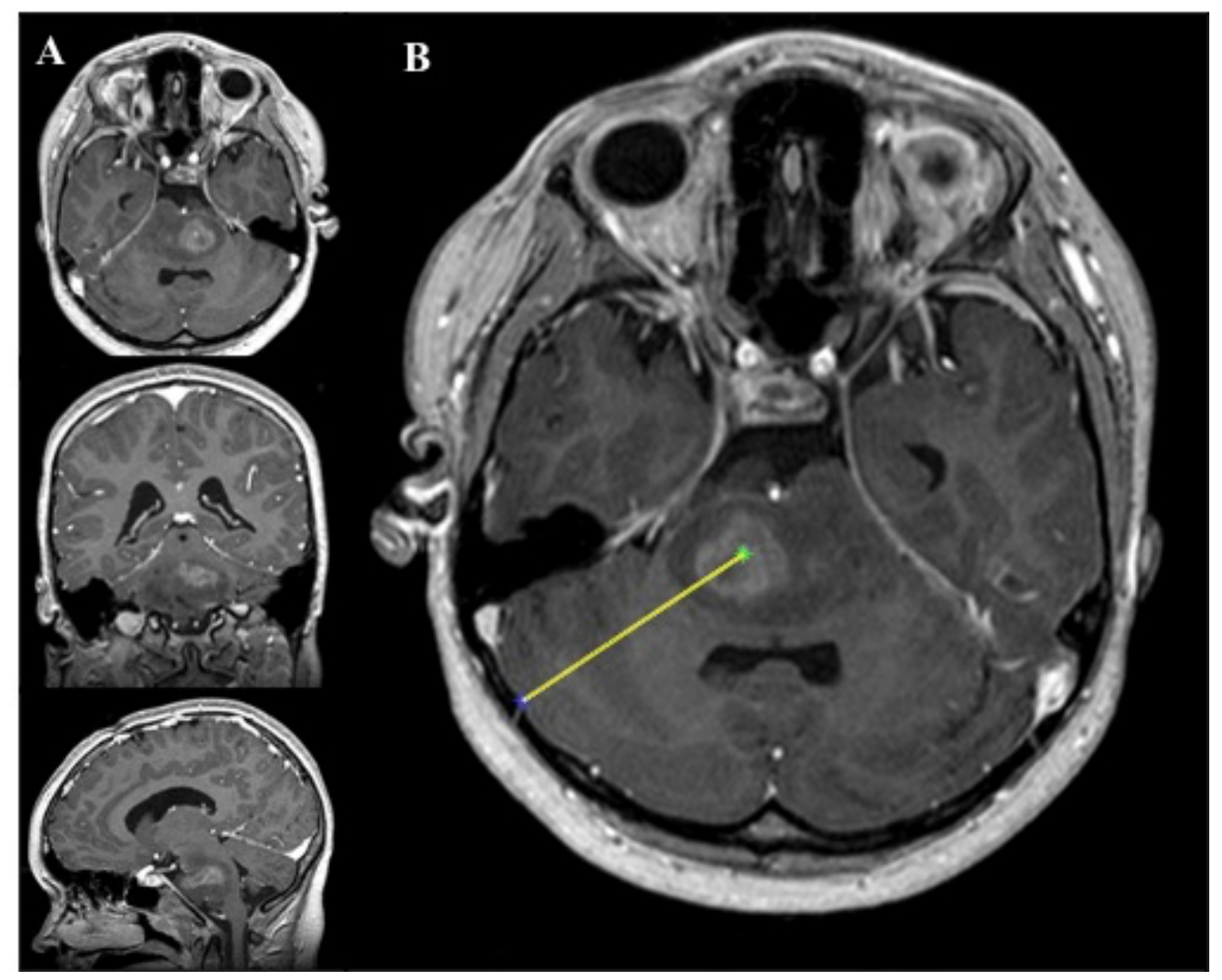

Figure 1

Case example of a transcerebellar trajectory for a pontine diffuse midline glioma: A: cerebral MRI with T1 gadolinium sequence in axial, coronal and sagittal views, B: trajectory axis view showing direct passage through the middle cerebellar peduncle. 


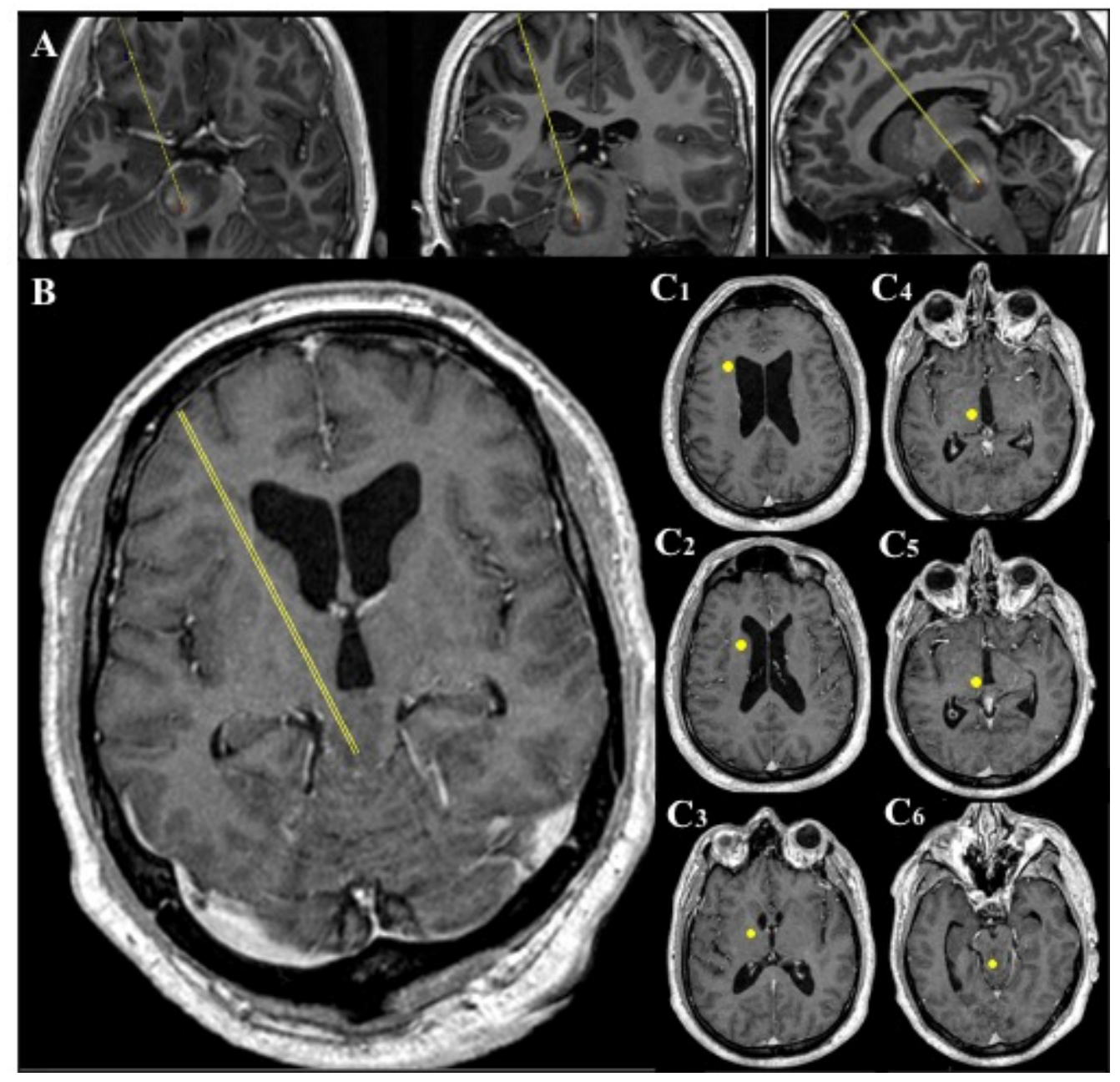

Figure 2

Case example of a transfrontal trajectory for a mesencephalic glioma : A: cerebral MRI with T1 gadolinium sequence in axial, coronal and sagittal views, B: trajectory axis view, C: axial view showing the transfrontal trajectory at multiple depths; yellow circle representing the trajectory's progression, from up to bottom and left column to right column: paraventricular (C1), lateral to the head of the caudate nucleus (C2), posterior to the anterior limb of the internal capsule through the internal globus pallidus (C3), passing the inferior and posterior limit of the thalamus to reach to mesencephalic lesion (C4 and C5), through the posterior part of the cerebral peduncle (C6). 


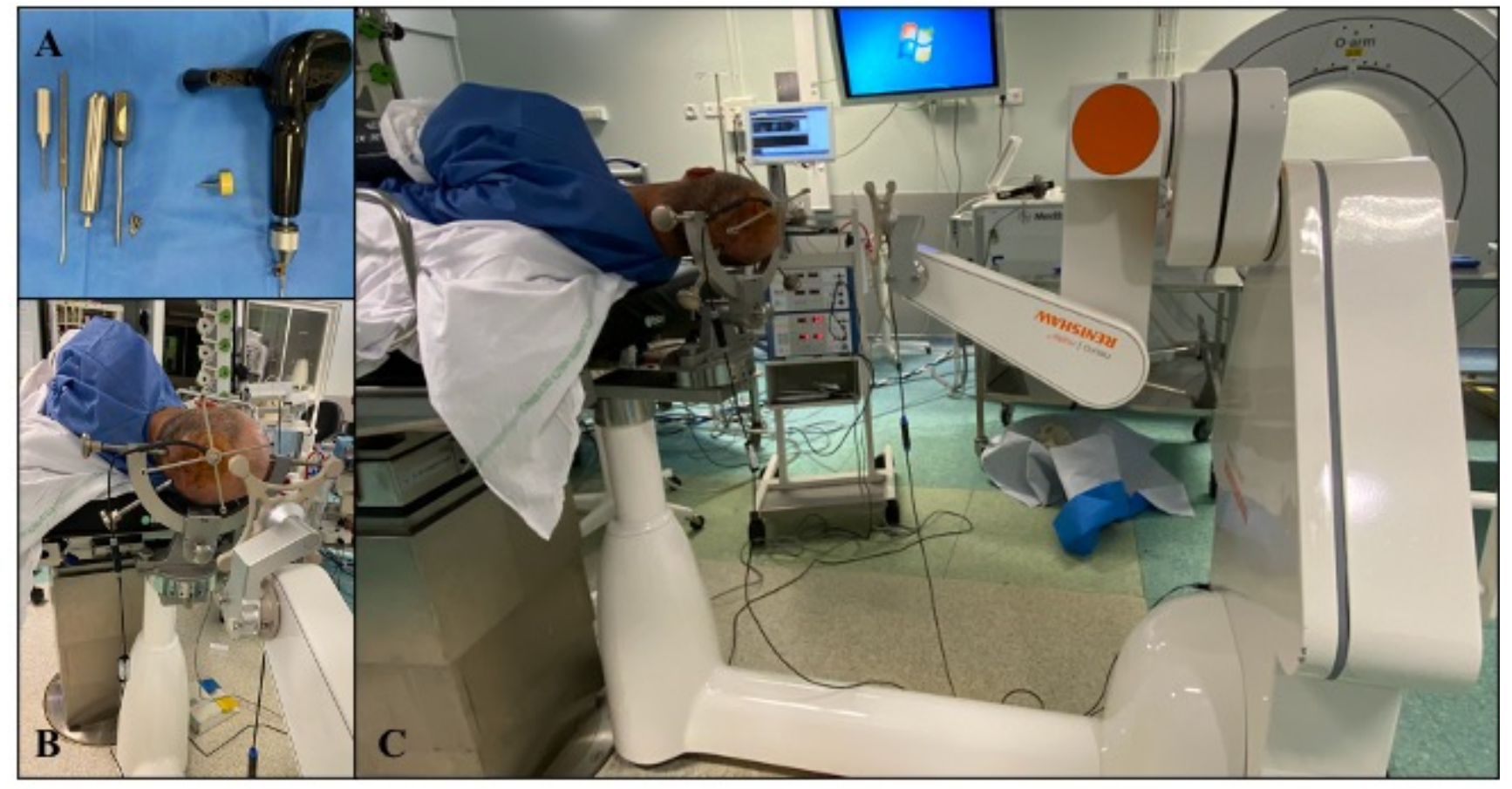

Figure 3

Intraoperative setting: A: fiducial marker instrumentation, B: ultrasound emitter system, C: patient positioning for transcerebellar approach 